\title{
Bile Duct Patency Maintained after Intraductal Radiofrequency Ablation in a Case of Hepatocellular Cholangiocarcinoma with Bile Duct Invasion
}

\author{
Sung Yong Han, Geun Am Song, Dong Uk Kim, Dong Hoon Baek, Moon Won Lee and Gwang Ha Kim \\ Department of Internal Medicine, Pusan National University Hospital, Biomedical Research Institute, and Pusan National University School \\ of Medicine, Busan, Korea
}

Combined hepatocellular-cholangiocarcinoma (HCC-CC) with bile duct invasion (BDI) is rare. In unresectable cases, biliary stent placement and photodynamic therapy (PDT) are used for resolving obstructive jaundice. However, stent occlusion remains problematic, and PDT is expensive and time-consuming. Intraductal radiofrequency ablation (RFA) is an emerging procedure for palliation in these patients. It has potential benefits including less expense, lower rates of severe complication, longer maintenance of ductal patency, and easier technique compared with PDT or stenting alone. We report a 67-year-old man who underwent repeated intraductal RFA for HCC-CC and HCC with BDI, for whom bile duct patency was maintained without additional biliary procedures. Clin Endosc 2018;51:201-205

Key Words: Carcinoma, hepatocellular; Intraductal ablation; Bile duct invasion

\section{INTRODUCTION}

Combined hepatocellular-cholangiocarcinoma (HCC-CC) is a rare primary liver cancer. ${ }^{1}$ Surgery is the treatment of choice, offering the possibility of a cure. Few cases have reported a favorable response to systemic chemotherapy or local treatment such as radiofrequency ablation (RFA) and transarterial chemoembolization (TACE).

HCC with bile duct invasion (HCC-BDI) is a rare condition with poor prognosis because of obstructive jaundice. Palliative methods may relieve jaundice, ensure quality of life, and possibly prolong survival. ${ }^{2-4}$ In patients with malignant bile duct

Received: June 12, 2017 Revised: June 29, 2017

Accepted: July 2, 2017

Correspondence: Geun Am Song

Division of Gastroenterology, Department of Internal Medicine, Pusan National University Hospital, 179 Gudeok-ro, Seo-gu, Busan 49241, Korea

Tel: +82-51-240-7869, Fax: +82-51-254-3237, E-mail: gasong@pusan.ac.kr ORCID: https://orcid.org/0000-0003-2100-8522

(c) This is an Open Access article distributed under the terms of the Creative Commons Attribution Non-Commercial License (http://creativecommons.org/ licenses/by-nc/3.0) which permits unrestricted non-commercial use, distribution, and reproduction in any medium, provided the original work is properly cited. obstruction, biliary stent placement and photodynamic therapy (PDT) commonly resolve obstructive jaundice. However, stent occlusion by tumor ingrowth or blood clots and recurrent cholangitis occur in patients with HCC ${ }^{5,6}$ Furthermore, PDT is expensive, time-consuming, and is associated with risks of complications, including hemobilia and photosensitivity. ${ }^{5}$ Therefore, safer and more effective methods for resolving HCC-BDI are needed. In recent literature, patients received intraductal RFA for obstructive jaundice caused by CC. Intraductal RFA has potential benefits including less expense, longer maintenance of ductal patency, and easier technique compared to PDT or stenting alone. ${ }^{7}$ HCC-BDI can be easily removed using an extraction balloon after RFA, since RFA induces coagulation necrosis of the HCC-BDI stalk connected with the main hepatic mass. Furthermore, intraductal RFA may help to maintain longer bile duct patency compared with that of stent placement or PDT.

We report a case of HCC-CC and HCC-BDI in a 67-yearold man with chronic hepatitis $\mathrm{B}$. He underwent repeated intraductal RFA for HCC-BDI, and maintained a patent bile duct without additional biliary procedures. 


\section{CASE REPORT}

A 67-year-old man presented with dyspepsia and epigastric discomfort, which had been present for about 6 weeks. After having an abdominal computed tomography (CT) scan performed at a local medical center, he presented to our hospital for evaluation of a mass at $\mathrm{S} 2 / 3$ and the left proximal intrahepatic bile duct (Fig. 1A). He had been diagnosed with chronic hepatitis B. The serum cancer antigen (CA) 19-9 level was $302.2 \mathrm{U} / \mathrm{mL}$ (reference range 0-39 U/mL), carcinoembryonic antigen level was $2.47 \mathrm{ng} / \mathrm{mL}$ (reference range $0-5.0 \mathrm{ng} / \mathrm{mL}$ ), and alpha-fetoprotein (AFP) level was $6.90 \mathrm{IU} / \mathrm{mL}$ (reference range $0-10 \mathrm{IU} / \mathrm{mL}$ ). A forceps biopsy was performed at the left intrahepatic bile duct mass during endoscopic retrograde cholangiopancreatography, resulting in chronic inflammation. The patient was pathologically diagnosed with CC based on percutaneous liver biopsy. Two intraductal RFA sessions were performed for the mass occupying the left proximal intrahepatic bile duct using a $2.2 \mathrm{~cm}$ RFA catheter (STARmed, Goyang, Korea) at the settings of $10 \mathrm{~W}$ for 120 seconds. During cholangiography, the left intrahepatic duct was not seen due to the suspicion of an intraductal mass (Fig. 2A). Necrotic materials were removed via the bile duct using an extraction balloon catheter after performing intraductal RFA (Fig. 2B, C). We ended the procedure after inserting an endoscopic nasobiliary drainage catheter for bleeding confirmation and irrigation purposes, following RFA. There was no definite bleeding. The necrotic materials were pathologically diagnosed as HCC, and the final pathologic diagnosis was HCC-CC and HCCBDI. After intraductal RFA and the sixth cycle of chemotherapy, the abdominal CT scan revealed the disappearance of the left intrahepatic ductal dilatation (Fig. 1B). It seemed that the
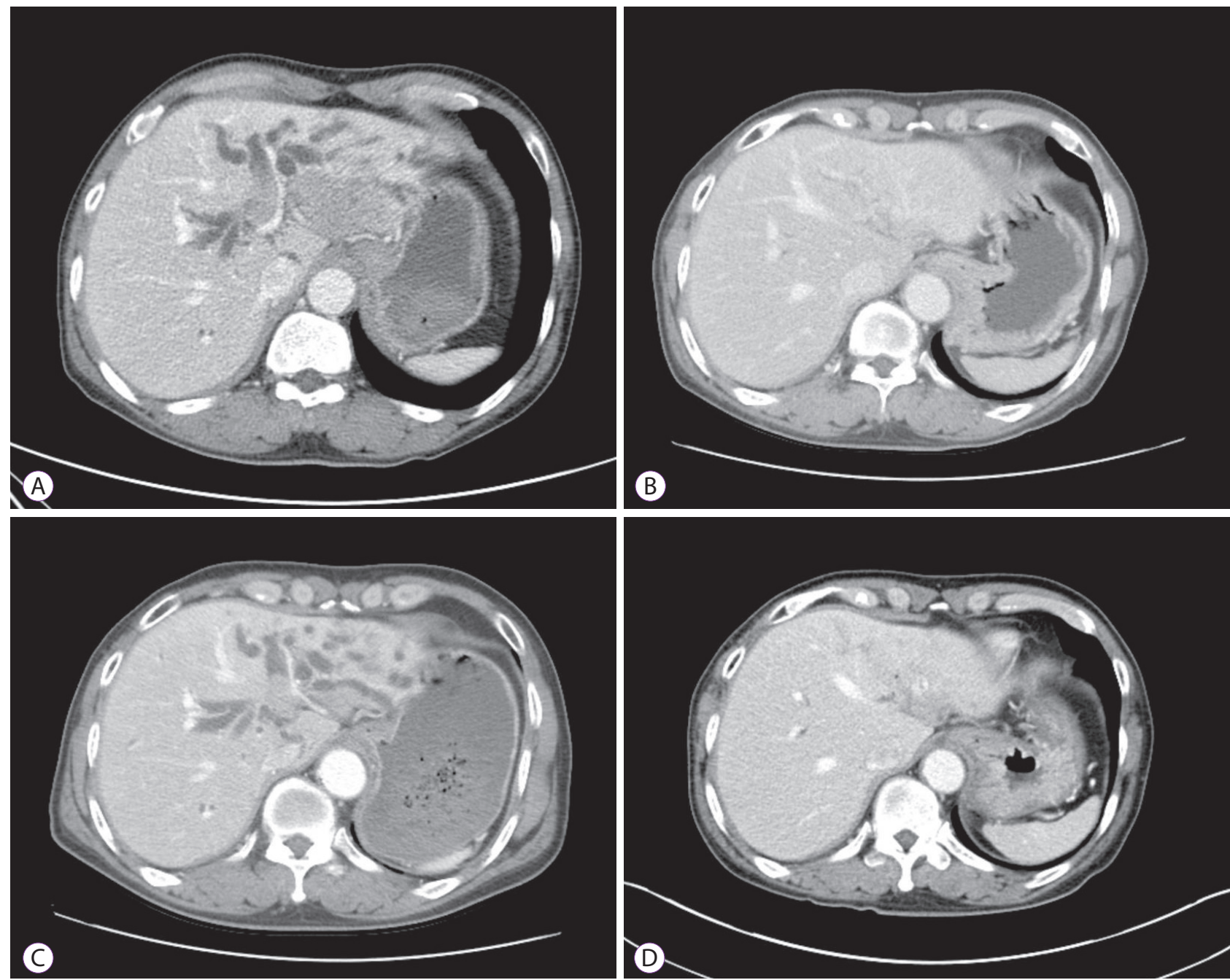

Fig. 1. Computed tomography (CT) image. (A) An initial CT revealed a 6.6-cm main mass at $S 2 / 3$ and a 2-cm intraductal mass at the left proximal intrahepatic bile duct. (B) A follow-up CT after 5 months revealed the disappearance of the left intrahepatic ductal dilatation after the first intraductal radiofrequency ablation (RFA) procedure and the sixth cycle of chemotherapy. (C) The next follow-up CT after 7 months revealed a recurrent intraductal mass with dilatation of the left intrahepatic bile duct. (D) A follow-up CT after 9 months revealed that the left intrahepatic bile duct dilatation was improved after repeated intraductal RFA. 
HCC-BDI was almost entirely removed by RFA. The patient underwent chemotherapy with gemcitabine $\left(1,000 \mathrm{mg} / \mathrm{m}^{2}\right)$ and cisplatin $\left(25 \mathrm{mg} / \mathrm{m}^{2}\right)$ on days 1 and 8 of a 21-day treatment cycle for CC and remnant HCC. After the sixth cycle of chemotherapy, the $6.6-\mathrm{cm}$ mass was reduced to $3.8 \mathrm{~cm}$. The serum CA 19-9 level was $6.20 \mathrm{U} / \mathrm{mL}$. Subsequently, the patient was hospitalized because of jaundice 6 months after intraductal RFA. An abdominal CT scan at that time revealed that the tumor size was decreased compared with the previous scan. However, the recurrence of the intraductal mass with dilatation of the left intrahepatic bile duct was observed (Fig. 1C). The serum CA 19-9 and AFP levels were rechecked. The CA
19-9 level increased to $106.2 \mathrm{U} / \mathrm{mL}$, and the AFP level was 4.71 $\mathrm{IU} / \mathrm{mL}$. We repeated the performance of intraductal RFA. Dilatation of the bile duct was decreased after intraductal RFA, as revealed by abdominal CT scan (Fig. 1D). The patient was discharged with the intent to treat with further chemotherapy.

\section{DISCUSSION}

In the current case, the patient was diagnosed as having HCC-CC and HCC-BDI. The main mass, an HCC-CC tumor located within segments II and III of the intrahepatic biliary
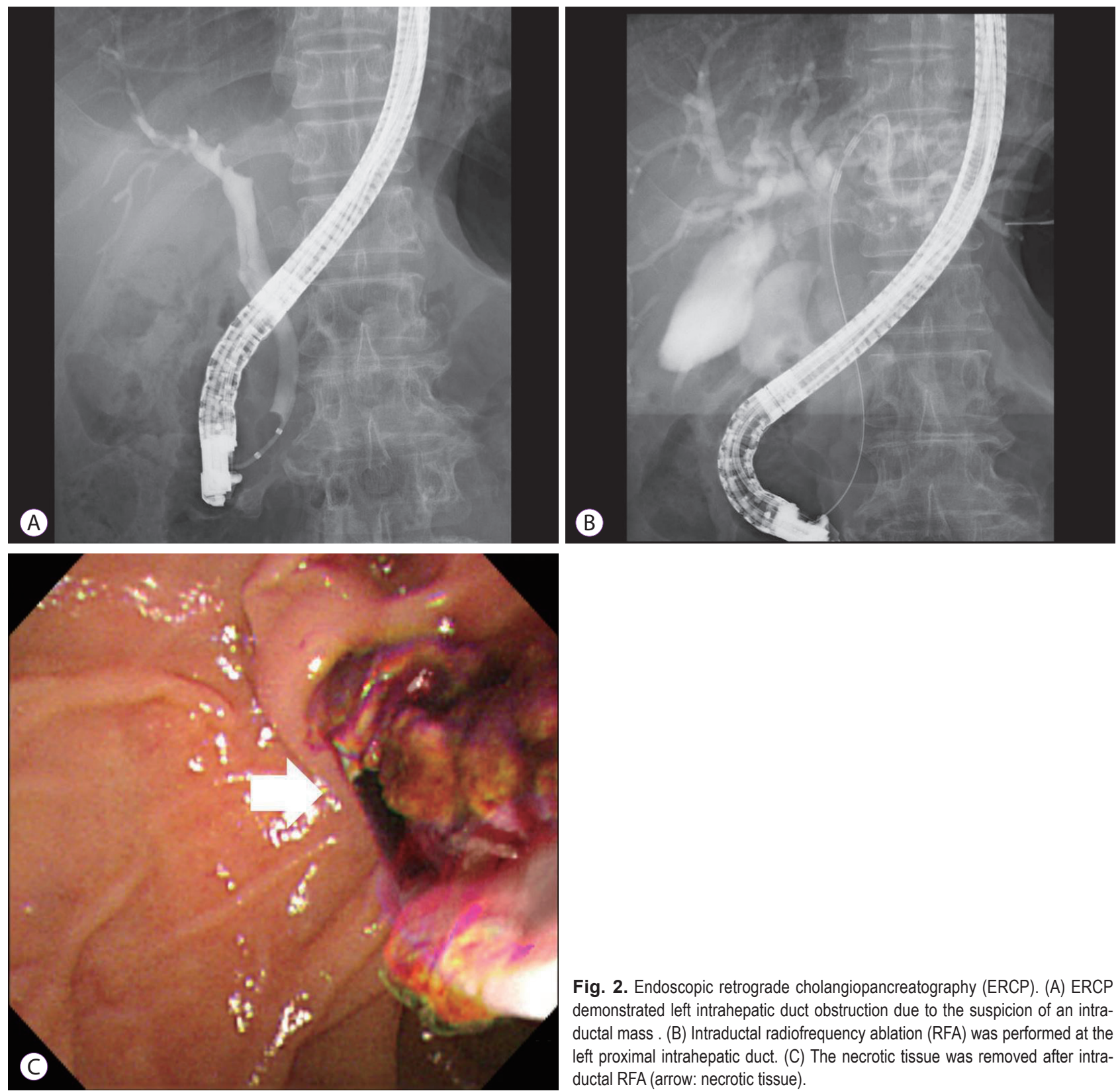

Fig. 2. Endoscopic retrograde cholangiopancreatography (ERCP). (A) ERCP demonstrated left intrahepatic duct obstruction due to the suspicion of an intraductal mass . (B) Intraductal radiofrequency ablation (RFA) was performed at the left proximal intrahepatic duct. (C) The necrotic tissue was removed after intraductal RFA (arrow: necrotic tissue). 
tree, appeared to respond favorably to systemic chemotherapy. In addition, the intraductal mass was thought to be HCC-BDI. The intraductal mass was easily removed using an extraction balloon, because intraductal RFA induces coagulation necrosis of the stalk. Bile duct patency was maintained after repeated intraductal RFA without additional procedures. Furthermore, there were no significant post-procedural complications.

HCC-CC is explained by some literature as follows: (1) it is a double primary cancer, (2) either HCC or CC arises first, and then transforms to other components; and (3) combined HCC-CC arises from intermediate cells, which differentiate into both components. ${ }^{8,9}$ HCC-CC is considered an aggressive tumor associated with poor prognosis with intermediate clinical features of both HCC and CC. HCC-CC tends to demonstrate portal and hepatic venous infiltration typical of HCC, and distant metastasis like that of $\mathrm{CC}^{8}{ }^{8}$ Surgical removal is the only treatment offering the possibility of a cure, although recurrence may be higher than that associated with pure HCC or CC. ${ }^{8,10}$ To our knowledge, there is no literature reporting the impact of local treatment options to improve survival of HCC-CC. Many HCC-CCs are less vascular and more fibrotic than HCC, and thus are less likely to respond to TACE or percutaneous ethanol injection. ${ }^{10}$ Therefore, RFA or cryoablation is useful in select patients with recurrent or unresectable tumors. ${ }^{10,11}$ If surgical intervention is not possible, local ablation treatment could possibly be used rather than conservative treatment.

HCC-BDI is a rare condition. HCC with obstructive jaundice has a poor prognosis (median survival 2-4 months, even with external drainage). ${ }^{2-4}$ The mechanism of HCC-BDI is not fully understood, but may be due to one of the following: (1) growth of a metastatic tumor along the bile duct, (2) intraductal growth of a metastatic tumor and separation of malignant cells from original malignant lesions, and (3) a necrotic and hemorrhagic tumor and blood clots occupying the bile duct. $^{6,7}$ Obstructive jaundice is the most relevant factor impacting survival and quality of life in patients suffering from bile duct malignancy. ${ }^{7}$ The most common method to resolve jaundice is the use of a biliary stent, and self-expandable metal stent (SEMS) placement is the standard palliative technique for patients with malignant bile duct obstruction. However, many patients with HCC-BDI have experienced hemobilia and recurrent cholangitis at the stenting site since tumor tissues are hypervascular and friable. Moreover, obstruction reportedly occurs due to tumor ingrowth after 6 months in most patients with a SEMS. ${ }^{5,6}$ In some cases, stent occlusion occurred earlier due to blood clots rather than tumor ingrowth. In cases of stent occlusion, PDT can be used to maintain ductal patency and improve overall survival. ${ }^{12,13}$ However, PDT is expensive and time-consuming. In addition to complications, including hemobilia of the stenting site and photosensitivity, one multicenter study demonstrated that patients who received PDT with stenting had a $60 \%$ increased mortality risk with poorer overall survival compared to that of stenting alone. ${ }^{14}$ A safer and more effective method to resolve HCC-BDI is therefore needed. Recent literature reported that patients received intraductal RFA for obstructive jaundice caused by CC. Hemobilia, cholangitis, bile duct injury, abscess formation and, in a few cases, mortality, were adverse events. ${ }^{5-7,15}$ Bile duct injury is one of the major complications of RFA and is mainly caused by thermal damage. When intraductal RFA is compared with percutaneous RFA, the incidence of bile duct injury is low because it is performed using low energy. Recently, one study showed that intraductal RFA is a safe and effective method in the animal model. ${ }^{16}$ Additionally, intraductal RFA for malignant biliary obstruction is technically easy. HCC-BDI is clinically distinct from $\mathrm{CC}$ in that obstructive jaundice in $\mathrm{CC}$ occurs by direct and broad invasion of the tumor into the bile duct, while in most patients with HCC, it occurs by indirect BDI of the tumor separated from the main mass. A bile duct tumor might attach to the bile duct mucosa with a thin stalk, without invasive growth into the submucosa. ${ }^{4}$ In our case, it was thought that the HCC-BDI tumor also had a thin stalk, because after intraductal RFA, removal of necrotic materials was easily performed. Furthermore, the absence of the intraductal mass was confirmed on the follow-up CT scan. General RFA has been used to treat small-sized HCC, ${ }^{17}$ and we posit that the technique could also serve as a therapeutic tool for HCC-BDI with a thin stalk. In patients with obstructive jaundice due to HCC-BDI, intraductal RFA may help maintain longer bile duct patency and improve overall survival compared with stent placement or PDT.

In conclusion, in the current case, biliary ductal patency was maintained for the 11 months of patient follow-up after repeated intraductal RFA, without need for additional procedures. We suggest that intraductal RFA may be an effective palliative method for maintaining ductal patency and improving overall survival, especially in patients with HCC-BDI.

Conflicts of Interest

The authors have no financial conflicts of interest.

\section{Acknowledgments}

This work was supported by a 2-Year Research Grant of Pusan National University.

\section{REFERENCES}

1. Garancini M, Goffredo P, Pagni F, et al. Combined hepatocellular-cholangiocarcinoma: a population-level analysis of an uncommon primary liver tumor. Liver Transpl 2014;20:952-959. 
2. Meng KW, Dong M, Zhang WG, Huang QX. Clinical characteristics and surgical prognosis of hepatocellular carcinoma with bile duct invasion. Gastroenterol Res Pract 2014;2014:604971.

3. Yu XH, Xu LB, Liu C, Zhang R, Wang J. Clinicopathological characteristics of 20 cases of hepatocellular carcinoma with bile duct tumor thrombi. Dig Dis Sci 2011;56:252-259.

4. Qin LX, Tang ZY. Hepatocellular carcinoma with obstructive jaundice: diagnosis, treatment and prognosis. World J Gastroenterol 2003;9:385391.

5. Rustagi T, Jamidar PA. Intraductal radiofrequency ablation for management of malignant biliary obstruction. Dig Dis Sci 2014;59:2635-2641.

6. Steel AW, Postgate AJ, Khorsandi S, et al. Endoscopically applied radiofrequency ablation appears to be safe in the treatment of malignant biliary obstruction. Gastrointest Endosc 2011;73:149-153.

7. Dolak W, Schreiber F, Schwaighofer H, et al. Endoscopic radiofrequency ablation for malignant biliary obstruction: a nationwide retrospective study of 84 consecutive applications. Surg Endosc 2014;28:854-860.

8. Chi M, Mikhitarian K, Shi C, Goff LW. Management of combined hepatocellular-cholangiocarcinoma: a case report and literature review. Gastrointest Cancer Res 2012;5:199-202.

9. Fujii H, Zhu XG, Matsumoto T, et al. Genetic classification of combined hepatocellular-cholangiocarcinoma. Hum Pathol 2000;31:1011-1017.

10. Wang J, Wang F, Kessinger A. Outcome of combined hepatocellular and cholangiocarcinoma of the liver. J Oncol 2010;2010.

11. Kassahun WT, Hauss J. Management of combined hepatocellular and cholangiocarcinoma. Int J Clin Pract 2008;62:1271-1278.

12. Ortner ME, Caca K, Berr F, et al. Successful photodynamic therapy for nonresectable cholangiocarcinoma: a randomized prospective study. Gastroenterology 2003;125:1355-1363.

13. Zoepf T, Jakobs R, Arnold JC, Apel D, Riemann JF. Palliation of nonresectable bile duct cancer: improved survival after photodynamic therapy. Am J Gastroenterol 2005;100:2426-2430.

14. Pereira SP, Aithal GP, Ragunath K, Devlin J, Owen F, Meadows H. Safety and long term efficacy of porfimer sodium photodynamic therapy in locally advanced biliary tract carcinoma. Photodiagnosis Photodyn Ther 2012;9:287-292.

15. Tal AO, Vermehren J, Friedrich-Rust M, et al. Intraductal endoscopic radiofrequency ablation for the treatment of hilar non-resectable malignant bile duct obstruction. World J Gastrointest Endosc 2014;6:13-19.

16. Cho JH, Lee KH, Kim JM, Kim YS, Lee DH, Jeong S. Safety and effectiveness of endobiliary radiofrequency ablation according to the different power and target temperature in a swine model. J Gastroenterol Hepatol 2017;32:521-526.

17. Künzli BM, Abitabile P, Maurer CA. Radiofrequency ablation of liver tumors: actual limitations and potential solutions in the future. World J Hepatol 2011;3:8-14. 